\title{
Gravitational Fields with Groups of Motions on Two-dimensional Transitivity Hypersurfaces in a Model with Matter and a Magnetic Field
}

\author{
I. S. SHIKIN \\ Moscow State University, Moscow, USSR
}

Received November 5, 1971

\begin{abstract}
For gravitational fields with metrics which admit of groups of motions multiply - transitive on 2-dimensional space-like invariant varieties, the exact solutions of the Einstein gravitational equations are given for the case when the sources of the gravitational field are dust-like matter and a magnetic field. A magnetic field is orientated along a direction orthogonal to transitivity hypersurface. The solutions contain arbitrary functions. In the case of transitivity hypersurface of positive curvature and in the absence of a magnetic field, the solution is reduced to the Tolman spherically symmetric solution for dust-like matter. The conditions are studied under which the solutions with a magnetic field become asymptotically isotropic and approach the flat and the open Friedmann models. The case of transitivity hypersurfaces with signature $(+-)$ is also considered.
\end{abstract}

\section{Introduction}

The paper deals with exact solutions of Einstein equations in General Relativity for metrics which admit of multiply transitive groups of motions on 2-dimensional transitivity hypersurfaces $V_{2}$. Exact solutions are given for the case when the sources of gravitational field are dust (incoherent matter) and a magnetic field, the direction of which is orthogonal to $V_{2}$. These solutions contain arbitrary functions. The cases considered are those in which the signature of $V_{2}$ is $(++)$ and $(+-)[1,2]$.

In the case of space-like $V_{2}$ with positive curvature and in the absence of a magnetic field these solutions are reduced to well known TolmanBondi solutions for spherically symmetric gravitational fields $[3,4]$.

A study of gravitational fields, the sources of which are matter and a magnetic field, is important in the theory of anisotropic cosmological models with a primordial magnetic field [5-10] and also is of interest for the problem of gravitational collapse in a magnetic field. Considered solutions include a homogeneous anisotropic model with euclidian co-moving space and also contain a class of inhomogeneous solutions which asymptotically become isotropic and approach the Friedmann solutions in the flat and in the open models. 
The general case of gravitational fields, whose metrics admit of 3-parameter groups of motions $G_{3}$ on $V_{2}$, is considered. Lie algebras $A_{3}$ of these groups allow extensions [11]. On the one hand, there exists a central extension of $A_{3}$ to $A_{4}$ with a space-like additional Killing vector. It leads to a group $G_{4}$ which acts on a hypersurface $V_{3}$, which is the direct topological product of $V_{2}$ and a line of a direction of a magnetic field. Such metrics have been considered previously [12,13,5-9]. They are consistent with the presence of a magnetic field. On the other hand, there exists noncentral extension of $A_{3}$ to $A_{4}$ which leads to a homogeneous anisotropic model with a co-moving 3-space of constant negative curvature. Also, there exist non-central extensions of $A_{3}$ to $A_{6}$, which lead to the Friedmann models. The non-central extensions, however, prove to be impossible in the case when a directed magnetic field serves as a source of gravitational field.

\section{Metrics with Groups of Motions on $V_{2}$}

Metrics which admit of 3-parameter groups of motions $G_{3}$, which act on space-like transitivity hypersurfaces $V_{2}$ with coordinates $x^{2}, x^{3}$, can be written in a synchronous system with geodesic lines of time $\tau\left(x^{0}=c \tau\right)$ in the form $[1,2,15]$

$$
\begin{gathered}
d s^{2}=(c d \tau)^{2}-d l^{2} \\
d l^{2}=X^{2}\left(x^{1}, \tau\right)\left(d x^{1}\right)^{2}+Y^{2}\left(x^{1}, \tau\right)\left[\left(d x^{2}\right)^{2}+f^{2}\left(x^{2}\right)\left(d x^{3}\right)^{2}\right] .
\end{gathered}
$$

$V_{2}$ is a 2-space of constant curvature, which may be zero, negative and positive, and the values in (2.1 a) respectively are

$$
\begin{aligned}
& f\left(x^{2}\right)=1, \\
& f\left(x^{2}\right)=\sinh x^{2}, \\
& f\left(x^{2}\right)=\sin x^{2} .
\end{aligned}
$$

Generators $X_{a}$ of a group of motions $G_{3}$ are defined by Killing vectors $\xi_{(a)}^{i}$ by $X_{a}=\xi_{(a)}^{i} \partial / \partial x^{i}$ (Latin indices $i, k, \ldots$ run from 0 to 3 ). For these operators the commutation relations $\left[X_{a}, X_{b}\right]=c_{a b}^{c} X_{c}$ are valid with structure constants which may be written in the form [14] (Latin indices $a, b, c$ run from 1 to $3 ; e_{a b c}$ is a skew pseudo-tensor, $e_{123}=1$ )

$$
c_{a b}^{c}=e_{a b d} n^{d c}+\delta_{b}^{c} a_{a}-\delta_{a}^{c} a_{b}, \quad n^{a b} a_{b}=0, \quad n^{a b}=n^{b a} .
$$

For (2.2a) the group $G_{3}$ is of type VII $_{0}$ according to Bianchi-Behr classification $[1,2,14,15]$ with the values in $(2.3)$

$$
a_{a}=0, \quad n^{a b}=\delta_{2}^{a} \delta_{2}^{b}+\delta_{3}^{a} \delta_{3}^{b},
$$


and with Killing vectors

$\xi_{(1)}^{i}=x^{3} \delta_{2}^{i}-x^{2} \delta_{3}^{i}, \quad \xi_{(2)}^{i}=\delta_{2}^{i}, \quad \xi_{(3)}^{i}=\delta_{3}^{i}, \quad X_{a}=\xi_{(a)}^{i} \partial / \partial x^{i}$.

For (2.2b) the group $G_{3}$ is of type VIII [2] with

$$
a_{a}=0, \quad n^{a b}=\delta_{1}^{a} \delta_{1}^{b}+\delta_{2}^{a} \delta_{2}^{b}-\delta_{3}^{a} \delta_{3}^{b},
$$

and Killing vectors are

$$
\xi_{(1)}^{i}=\cos x^{3} \delta_{2}^{i}-\sin x^{3} \operatorname{coth} x^{2} \delta_{3}^{i}, \quad \xi_{(2)}^{i}=\partial \xi_{(1)}^{i} / \partial x^{3}, \quad \xi_{(3)}^{i}=\delta_{3}^{i} .
$$

For (2.2c) the group $G_{3}$ belongs to type IX [2] with

$$
a_{a}=0, \quad n^{a b}=\delta_{1}^{a} \delta_{1}^{b}+\delta_{2}^{a} \delta_{2}^{b}+\delta_{3}^{a} \delta_{3}^{b},
$$

and Killing vectors are

$$
\xi_{(1)}^{i}=\cos x^{3} \delta_{2}^{i}-\sin x^{3} \cot x^{2} \delta_{3}^{i}, \quad \xi_{(2)}^{i}=\partial \xi_{(1)}^{i} / \partial x^{3}, \quad \xi_{(3)}^{i}=\delta_{3}^{i} .
$$

Lie algebras $A_{3}$ of generators of the groups $G_{3}(2.4),(2.5),(2.6)$ may be extended $[11,16,17]$. Central extension is carried out by addition of the generator $X_{4}$ which commutes with the generators of $A_{3}$, whereas for non-central extensions the generators, which are added, do not commute with the generators of $A_{3}$. A central extension is given by addition a Killing vector (with $\xi_{(4)}^{2}=\xi_{(4)}^{3}=0$ ), space-time character of which may be arbitrary [11]. A time-like additional Killing vector would lead to static solutions which contain in particular the Reissner-Nordstrom metric. We confine ourselves to a central extension given by a spacelike additional Killing vector

$$
\xi_{(4)}^{i}=\delta_{1}^{i}, \quad X_{4}=\partial / \partial x^{1},
$$

which leads in (2.1a) to

$$
X=b(\tau), \quad Y=a(\tau)
$$

for all possible values of $f\left(x^{2}\right)(2.2 \mathrm{a}),(2.2 \mathrm{~b}),(2.2 \mathrm{c})[5-7,9,12,13,18]$.

For $f\left(x^{2}\right)=1$ this central extension leads to the group $G_{4}$ which in virtue of (2.4a) and (2.7) contains the abelian subgroup $G_{3}$ of $X_{2}, X_{3}, X_{4}$. This subgroup $G_{3}$ is simply transitive on 3-dimensional transitivity hypersurface $x^{1}, x^{2}, x^{3}$ and belongs to Bianchi type I with $a^{a}=0, n^{a b}=0$ in (2.3). Such model is of interest in anisotropic cosmology $[1,19]$. Lie algebra $A_{4}$ with (2.8) for $f\left(x^{2}\right)=1$ is extended to $A_{6}$ by addition the Killing vectors

$$
\xi_{(5)}^{i}=x^{3} \delta_{1}^{i}-x^{1} \delta_{3}^{i}, \quad \xi_{(6)}^{i}=x^{1} \delta_{2}^{i}-x^{2} \delta_{1}^{i} .
$$

It leads in (2.1a) to $X=Y=a(\tau)$ and yields the flat Friedmann model. For $f\left(x^{2}\right)=\sinh x^{2}$ the central extension of (2.5a) by (2.7), (2.8) leads to the group $G_{4}$ which contains subgroup $G_{3}$ (with $2 X_{1},\left(X_{2}+X_{3}+X_{4}\right) / 2$, $\left.\left(X_{2}+X_{3}-X_{4}\right) / 2\right)$, which is simply transitive on 3-dimensional space 
$x^{1}, x^{2}, x^{3}$ and is of Bianchi type III with $a^{a}=-\delta_{1}^{a}, n^{a b}=\delta_{2}^{a} \delta_{2}^{b}-\delta_{3}^{a} \delta_{3}^{b}$ in (2.3) [20].

In both cases (2.2b) and (2.2c) Lie algebras $A_{4}$ of the groups $G_{4}$ with (2.8) do not allow a further extension to algebras $A_{6}$, so (2.1a), (2.8) with (2.2b) and (2.2c) can not be reduced by a further specialization to the metrics of isotropic Friedmann models.

Consider non-central extensions of $A_{3}$. For $f\left(x^{2}\right)=1$ there exists the non-central extension of algebra $A_{3}(2.4 \mathrm{a})$ to $A_{4}$ which is given by addition the Killing vector

$$
\xi_{(4)}^{i}=\delta_{1}^{i}+x^{2} \delta_{2}^{i}+x^{3} \delta_{3}^{i}, \quad X_{4}=\xi_{(4)}^{i} \partial / \partial x^{i} .
$$

It leads to the group $G_{4}$ [21] of the type $G_{4} \mathrm{~V}$ according to Petrov [2], which contains the subgroup $G_{3}$ of $X_{4}, X_{2}, X_{3}$. This subgroup is simply transitive on 3-space $x^{1}, x^{2}, x^{3}$ and is of Bianchi type $\mathrm{V}$ with $a^{a}=-\delta_{1}^{a}$, $n^{a b}=0$ in (2.3). The extension (2.9) gives in (2.1 a)

$$
X=b(\tau), \quad Y\left(x^{1}, \tau\right)=\exp \left(-x^{1}\right) a(\tau) ; \quad f\left(x^{2}\right)=1 .
$$

Algebra $A_{4}(2.4 \mathrm{a}),(2.9)$ is extended to $A_{6}$ by addition the Killing vectors

$$
\begin{aligned}
& \xi_{(5)}^{i}=2 x^{2} \delta_{1}^{i}+\left[\left(x^{2}\right)^{2}-\left(x^{3}\right)^{2}-\exp \left(2 x^{1}\right)\right] \delta_{2}^{i}+2 x^{2} x^{3} \delta_{3}^{i}, \\
& \xi_{(6)}^{i}=2 x^{3} \delta_{1}^{i}+2 x^{2} x^{3} \delta_{2}^{i}+\left[-\left(x^{2}\right)^{2}+\left(x^{3}\right)^{2}-\exp \left(2 x^{1}\right)\right] \delta_{3}^{i},
\end{aligned}
$$

and it leads to the open Friedmann model in (2.1a) with

$$
X=a(\tau), \quad Y=\exp \left(-x^{1}\right) a(\tau) ; \quad f\left(x^{2}\right)=1 .
$$

For $f\left(x^{2}\right)=\sinh x^{2}$ a non-central extension of $A_{3}(2.5 \mathrm{a})$ to $A_{4}$ is impossible. There exists the non-central extension of (2.5a) to $A_{6}$ given by addition

$$
\begin{aligned}
& \quad \xi_{(4)}^{i}=\cosh x^{2} \delta_{1}^{i}-\tanh x^{1} \sinh x^{2} \delta_{2}^{i}, \\
& \xi_{(5)}^{i}=\sin x^{3} \sinh x^{2} \delta_{1}^{i}-\tanh x^{1} \sin x^{3} \cosh x^{2} \delta_{2}^{i}-\frac{\tanh x^{1} \cos x^{3}}{\sinh x^{2}} \delta_{3}^{i}, \\
& \xi_{(6)}^{i}=\frac{\partial \xi_{(5)}^{i}}{\partial x^{3}} .
\end{aligned}
$$

It leads to the open Friedmann model in (2.1a) with

$$
X=a(\tau), \quad Y=\cosh x^{1} a(\tau) ; \quad f\left(x^{2}\right)=\sinh x^{2} .
$$

For $f\left(x^{2}\right)=\sin x^{2}$ there exist non-central extensions of (2.6a) to $A_{6}$ which yield the Friedmann metrics in the flat, the open and the closed models in a usual form [3] respectively with the values in (2.1a)

$X=a(\tau), \quad Y=x^{1} a(\tau), \quad \sinh x^{1} a(\tau), \quad \sin x^{1} a(\tau) ; \quad f\left(x^{2}\right)=\sin x^{2}$.

Formulae (2.12) are well-known while the expressions (2.10) and (2.11) for the open Friedmann model are more unusual. 
Now consider Einstein's field equations (without $\Lambda$-term)

$$
R_{i}^{k}-(R / 2) \delta_{i}^{k}=\left(8 \pi k / c^{4}\right) T_{i}^{k}
$$

for the metric (2.1), (2.1a) (the notations are those of [3]). In terms of $\partial / \partial \tau={ }^{\prime} ; \partial / \partial x^{1}=^{\prime} ; h_{1}=\dot{X} / X, h_{2}=\dot{Y} / Y, \lambda_{1}=X^{\prime} / X, \lambda_{2}=Y^{\prime} / Y$, the non-zero components of (2.13) become

$$
\begin{aligned}
R_{0}^{0}- & (R / 2) \\
= & \frac{1}{c^{2}} h_{2}\left(h_{2}+2 h_{1}\right)+\frac{1}{X^{2}}\left(-2 \lambda_{2}^{\prime}+2 \lambda_{1} \lambda_{2}-3 \lambda_{2}^{2}\right)-\frac{1}{Y^{2}} \frac{d^{2} f}{f\left(d x^{2}\right)^{2}} \\
= & \frac{8 \pi k}{c^{4}} T_{0}^{0} ; \\
R_{1}^{1}-\frac{R}{2}= & \frac{1}{c^{2}}\left(2 \dot{h}_{2}+3 h_{2}^{2}\right)-\frac{1}{X^{2}} \lambda_{2}^{2}-\frac{1}{Y^{2}} \frac{d^{2} f}{f\left(d x^{2}\right)^{2}}=\frac{8 \pi k}{c^{4}} T_{1}^{1} ;(2.15 b \\
R_{2}^{2}-(R / 2) & =R_{3}^{3}-(R / 2) \\
& =\frac{1}{c^{2}}\left(\dot{h}_{1}+\dot{h}_{2}+h_{1}^{2}+h_{2}^{2}+h_{1} h_{2}\right)+\frac{1}{X^{2}}\left(-\lambda_{2}^{\prime}+\lambda_{1} \lambda_{2}-\lambda_{2}^{2}\right) \\
& =\frac{8 \pi k}{c^{4}} T_{2}^{2} ; \\
R_{1}^{0} & =\frac{2}{c}\left[\lambda_{2}\left(h_{1}-h_{2}\right)-h_{2}^{\prime}\right]=\frac{8 \pi k}{c^{4}} T_{1}^{0} ; \quad R_{2}^{0}=R_{3}^{0}=0 .
\end{aligned}
$$

The non-zero components of Rucci 3-tensor in the space (2.1a) are

$$
\begin{aligned}
& P_{1}^{1}=\frac{2}{X^{2}}\left[-\lambda_{2}^{\prime}+\lambda_{2}\left(\lambda_{1}-\lambda_{2}\right)\right], \\
& P_{2}^{2}=P_{3}^{3}=\frac{1}{X^{2}}\left[-\lambda_{2}^{\prime}+\lambda_{2}\left(\lambda_{1}-2 \lambda_{2}\right)\right]-\frac{d^{2} f}{Y^{2} f\left(d x^{2}\right)^{2}} .
\end{aligned}
$$

Weyl tensor for the metric (2.1), (2.1a) in a general case is of type $D$; in special cases (2.10), (2.11), (2.12) it is reduced to zero.

In the cases when the signature of transitivity hypersurfaces $V_{2}$ is $(+-)$, two forms for an interval are possible

$$
\begin{aligned}
d s^{2}= & Y^{2}\left(x^{2}, x^{3}\right)\left[\left(d x^{0}\right)^{2}-f^{2}\left(x^{0}\right)\left(d x^{1}\right)^{2}\right] \\
& -X^{2}\left(x^{2}, x^{3}\right)\left(d x^{2}\right)^{2}-\left(d x^{3}\right)^{2}, \\
d s^{2}= & Y^{2}\left(x^{2}, x^{3}\right)\left[f^{2}\left(x^{1}\right)\left(d x^{0}\right)^{2}-\left(d x^{1}\right)^{2}\right] \\
& -X^{2}\left(x^{2}, x^{3}\right)\left(d x^{2}\right)^{2}-\left(d x^{3}\right)^{2}
\end{aligned}
$$

with the functions (2.2a), (2.2b), (2.2c) of the respective arguments. 
For $f=1$ in (2.17a), (2.17b) the group $G_{3}$ [2] is of type $\mathrm{VI}_{0}$ of BianchiBehr classification with the values in (2.3) and with Killing vectors given by

$a_{a}=0, n^{a b}=\delta_{1}^{a} \delta_{1}^{b}-\delta_{2}^{a} \delta_{2}^{b} ; \xi_{(1)}^{i}=\delta_{0}^{i}, \xi_{(2)}^{i}=\delta_{1}^{i}, \xi_{(3)}^{i}=x^{1} \delta_{0}^{i}+x^{0} \delta_{1}^{i}$.

In the case $f(y)=\sinh y$ in (2.17a), (2.17b) $G_{3}$ is of type VIII with (2.5) and Killing vectors for $(2.17 \mathrm{a})$ are given by

$\xi_{(1)}^{i}=\cosh x^{1} \delta_{0}^{i}-\sinh x^{1} \operatorname{coth} x^{0} \delta_{1}^{i}, \quad \xi_{(2)}^{i}=\delta_{1}^{i}, \quad \xi_{(3)}^{i}=\partial \xi_{(1)}^{i} / \partial x^{1}$.

For $f(y)=\sin y$ in (2.17a), (2.17b) $G_{3}$ is also of type VIII and for (2.17a)

$\xi_{(1)}^{i}=\cosh x^{1} \delta_{0}^{i}-\sinh x^{1} \cot x^{0} \delta_{1}^{i}, \quad \xi_{(2)}^{i}=\delta_{1}^{i}, \quad \xi_{(3)}^{i}=\partial \xi_{(1)}^{i} / \partial x^{1}$.

Killing vectors for (2.17b) are given by (2.19), (2.20) with the permutation of $x^{0}$ with $x^{1}$ and of $\xi^{0}$ with $\xi^{1}$.

The central extension of $A_{3}$ for (2.17a), (2.17b) is given by addition the Killing vector $\xi_{(4)}^{i}=\delta_{2}^{i}$. It leads in (2.17a), (2.17b) to

$$
X=X\left(x^{3}\right), \quad Y=Y\left(x^{3}\right) .
$$

Weyl tensor $C_{i k l m}$ for the metrics (2.17a), (2.17b) is also of type $D$ with the equal eigenvalues of $C_{0 \alpha 0 \beta}\left(C_{0 \alpha \beta \gamma}=0\right)$ in the orthonormal tetrad in $x^{2}, x^{3}$ directions.

\section{Gravitational Fields in a Model with Dust and a Magnetic Field}

We shall consider solutions of the Einstein equations under assumption that the energy-momentum tensor in (2.13) is the superposition of that of dust-like matter and that of electromagnetic field:

$$
T_{i}^{k}=\left(T_{i}^{k}\right)^{\mathrm{I}}+\left(T_{i}^{k}\right)^{\mathrm{II}} .
$$

The energy-momentum tensor for dust (pressure-free matter) is

$$
\left(T_{i}^{k}\right)^{\mathrm{I}}=e u_{i} u^{k}, \quad u_{i} u^{i}=1,
$$

where $u^{i}$ is the 4-velocity and $e=\varrho c^{2}$ is the energy density of matter. The invariance of the metric leads to the invariance of the 4-velocity in (2.13), (3.2) under transformations of the groups $G_{3}(2.4),(2.5),(2.6)$, so the operator $u^{i} \partial / \partial x^{i}$ must commute with the generators of the groups. It gives for (2.1), (2.1a)

$$
u^{i}=u^{0}\left(x^{1}, \tau\right) \delta_{0}^{i}+u^{1}\left(x^{1}, \tau\right) \delta_{1}^{i},
$$


and in the case of the extensions with (2.8) and (2.9a)

$$
u^{0}=u^{0}(\tau), \quad u^{1}=u^{1}(\tau) .
$$

The conservation laws are

$$
\left(T_{i}^{k}\right)_{; k}=0 .
$$

We shall assume for the electromagnetic field that the Lorentz 4-force is reduced to zero i.e.

$$
\left(T_{i}^{k}\right)_{; k}^{\mathrm{II}}=0 .
$$

For dust Eqs. (3.5) in virtue of (3.5a) give the continuity equation

$$
u^{i}\left(T_{i}^{k}\right)_{; k}^{\mathbf{I}}=\left(e u^{k}\right)_{; k}=0,
$$

and the equations of momentum

$$
\left(T_{i}^{k}\right)_{; k}^{\mathrm{I}}=e u^{k}\left(u_{i}\right)_{; k}=0,
$$

which mean that the world lines of dust are geodesic.

Eq. (3.7) together with $u^{i} u_{i ; k}=0$ lead to the equation for vorticity

$$
u^{i} \omega_{i k}=0, \quad \omega_{i k}=u_{i ; k}-u_{k ; i},
$$

which is reduced under condition (3.3) to

$$
\omega_{i k}=0, \quad u_{i}=\partial \varphi\left(x^{0}, x^{1}\right) / \partial x^{i} .
$$

Thus, in the case under consideration a motion of matter is irrotational. On account of this the system of coordinates (2.1), (2.1a) can be transformed into the synchronous and comoving system by means of the transformation

$$
\left(x^{0}\right)^{\prime}=\varphi\left(x^{0}, x^{1}\right), \quad u^{m} \partial\left(x^{\alpha}\right)^{\prime} / \partial x^{m}=0, \quad \alpha=1,2,3 .
$$

In such a system the geodesic lines $\tau$ are world lines of matter.

In the further consideration with groups $G_{3}$ we imply that the system (2.1), (2.1a) is co-moving, so in (3.3), (3.2)

$$
u^{0}=u_{0}=1, \quad u^{1}=0 ; \quad\left(T_{i}^{k}\right)^{\mathrm{I}}=e \delta_{i}^{0} \delta_{0}^{k} .
$$

Electromagnetic field can be described in terms of space-like 4-vectors $h^{i}$ and $e^{i}$ which are defined through the electromagnetic field tensor $F_{i k}$ and the 4-velocity $u^{i}$ by $[22,23]$

$$
\begin{gathered}
h_{k}=\frac{1}{2} E_{i k l m} u^{i} F^{l m}, \quad e_{k}=u^{i} F_{i k}, \quad h^{i} u_{i}=e^{i} u_{i}=0, \\
h^{i} h_{i}=-|h|^{2}, \quad e^{i} e_{i}=-|e|^{2} ; \quad F_{i k}=u_{i} e_{k}-u_{k} e_{i}-E_{i k l m} u^{l} h^{m},
\end{gathered}
$$

where $E_{i k l m}=e_{i k l m}(-g)^{1 / 2}, e_{i k l m}$ is a skew pseudo-tensor with $e^{0123}=1$. The energy-momentum tensor of electromagnetic field is written in 
terms of $h^{i}, e^{i}$ and $u^{i}$ by

$$
\begin{aligned}
\left(T_{i}^{k}\right)^{\mathrm{II}} & =\frac{1}{4 \pi}\left[\left(u_{i} u^{k}-\frac{1}{2} \delta_{i}^{k}\right)\left(|h|^{2}+|e|^{2}\right)-h_{i} h^{k}-e_{i} e^{k}-u_{i} v^{k}-u^{k} v_{i}\right], \\
v_{i} & =E_{i k l m} e^{k} h^{l} u^{m} .
\end{aligned}
$$

Maxwell equations with aid of (3.8) are written

$$
\begin{gathered}
\left(u^{i} h^{k}-u^{k} h^{i}+E^{i k l m} u_{l} e_{m}\right)_{; k}=0, \\
F_{; k}^{i k} \equiv\left(u^{i} e^{k}-u^{k} e^{i}-E^{i k l m} u_{l} h_{m}\right)_{; k}=-4 \pi j^{i} / c .
\end{gathered}
$$

The invariance of the metric (2.1), (2.1a) under the groups of motions $G_{3}$ leads to the invariance of the vectors $h^{i}$ and $e^{i}$ in (3.8a) under these groups. It yields similarly to (3.3)

$$
h^{i}=h^{0}\left(x^{1}, \tau\right) \delta_{0}^{i}+h^{1}\left(x^{1}, \tau\right) \delta_{1}^{i}, \quad e^{i}=e^{0}\left(x^{1}, \tau\right) \delta_{0}^{i}+e^{1}\left(x^{1}, \tau\right) \delta_{1}^{i} .
$$

In the case of the co-moving system (2.1), (2.1a) with (3.3a) the orthogonality conditions (3.8) lead to

$$
h^{i}=h^{1}\left(x^{1}, \tau\right) \delta_{1}^{i}, \quad e^{i}=e^{1}\left(x^{1}, \tau\right) \delta_{1}^{i} .
$$

Consideration of $F_{i k}$ with (3.11) in an orthonormal frame yields

$$
|h|^{2}=X^{2}\left(h^{1}\right)^{2}=H^{2}, \quad|e|^{2}=X^{2}\left(e^{1}\right)^{2}=E^{2} .
$$

It shows that in the co-moving system (2.1), (2.1a) there exist the collinear magnetic and electric fields, which are directed along $x^{1}$ and have intensities $H$ and $E$.

Maxwell equations (3.10) show that the 4-current $j^{i}$ is equal to zero in accordance with (3.5a) whereas (3.9) for (3.11) gives

$$
\begin{gathered}
\frac{h_{1}}{X}=H=\frac{K_{1}}{Y^{2}}, \quad \frac{e_{1}}{X}=E=\frac{K_{2}}{Y^{2}}, \quad K_{1}=\text { const }, \\
K_{2}=\text { const } ; \quad e^{i}=\frac{K_{2}}{K_{1}} h^{i} .
\end{gathered}
$$

Non-zero components of (3.8a) in virtue of (3.12) are given by

$$
\begin{gathered}
\left(T_{0}^{0}\right)^{\mathrm{II}}=\left(T_{1}^{1}\right)^{\mathrm{II}}=-\left(T_{2}^{2}\right)^{\mathrm{II}}=-\left(T_{3}^{3}\right)^{\mathrm{II}}=W=\frac{H^{2}+E^{2}}{8 \pi}=\frac{K^{2}}{8 \pi Y^{4}}, \\
K^{2}=K_{1}^{2}+K_{2}^{2}=\text { const } .
\end{gathered}
$$

In the case of the central extension of $A_{3}$ to $A_{4}$ with (2.8) the Eq. (2.15d) yields $R_{01}=0$. It shows due to (3.8a), (3.2) that in this case also $u^{1}=0$, so (3.3a) and (3.12), (3.12a) with $H=H(\tau), E=E(\tau)$ remain valid. 
For the metric (2.1), (2.1a) with (3.3a) one obtains

$$
c u_{; k}^{i}=h_{1} \delta_{1}^{i} \delta_{k}^{1}+h_{2}\left(\delta_{2}^{i} \delta_{k}^{2}+\delta_{3}^{i} \delta_{k}^{3}\right),
$$

so a motion of matter is described by expansion with the scalar $\Theta=\left(h_{1}+2 h_{2}\right) / 3$ and by shear for $h_{1} \neq h_{2}$ while rotation is absent.

Now consider the integration of the Einstein gravitational equations (2.15), (3.1) in the co-moving synchronous system (2.1), (2.1a) for dust with (3.3a) and electromagnetic field with (3.12), (3.12a). The case when both a magnetic and an electric field are present and the case when only a magnetic field is present are distinguished in (3.12a) merely by the value of a constant $K$. For the sake of simplicity we shall speak only of the presence of a magnetic field, which is physically reasonable for many problems.

The continuity Eq. (3.6) gives

In the case

$$
e=\Psi\left(x^{1}\right) / X Y^{2} .
$$

$$
\lambda_{2}=0, \quad Y=Y(\tau),
$$

the Eq. $(2.15 \mathrm{~d})$ (with $\left.T_{1}^{0}=0\right)$ is satisfied identically. In this case Eqs (2.15a) and (2.15c) with (3.12a) lead to the relation $X=\varphi_{1}\left(x^{1}\right) X(\tau)$ which can be written with the aid of possible transformations of $x^{1}$ in the form

$$
X=X(\tau) .
$$

Thus, $\lambda_{2}=0$ corresponds to the group $G_{4}$ with (2.7). The result of the integration of the Einstein field equations for dust and a magnetic field under conditions (3.14), (3.14a) is given in [7] (also [6,18]). It must be pointed out that this solution becomes isotropic asymptotically as $|\tau| \rightarrow \infty$ only for the value $f\left(x^{2}\right)=1$, this being in agreement with the possibility in this case of the extension of $A_{4}$ to $A_{6}$, which corresponds to the flat Friedmann model. The solution of the Einstein equations for (3.14), (3.14a) in the case when only matter is present is considered in $[12,13,24]$.

The condition $\lambda_{2} \neq 0$ corresponds to a group $G_{3}$ for (2.1), (2.1a) (and to the non-central extensions). In this case Eq. (2.15d) gives

$$
X=\psi\left(x^{1}\right) Y^{\prime} .
$$

We shall use an arbitrary function $\varphi\left(x^{1}\right)$ defined by

where

$$
\begin{aligned}
& 1 / \psi^{2}\left(x^{1}\right)= \pm \alpha^{2}+\varphi\left(x^{1}\right), \\
& \mp \alpha^{2}=d^{2} f\left(x^{2}\right) / f\left(d x^{2}\right)^{2}
\end{aligned}
$$

with the functions (2.2a), (2.2b), (2.2c), so $\alpha^{2}=0$ for (2.2a), $\alpha^{2}=1$ with the lower sign for (2.2b) and $\alpha^{2}=1$ with the upper sign for (2.2c). Eqs. (3.15a) 
and (3.16) lead to the inequalities

$$
\begin{aligned}
& f\left(x^{2}\right)=1: \quad \varphi\left(x^{1}\right)>0, \\
& f\left(x^{2}\right)=\sinh x^{2}: \varphi\left(x^{1}\right)>1, \\
& f\left(x^{2}\right)=\sin x^{2}: \quad \varphi\left(x^{1}\right)+1>0 .
\end{aligned}
$$

Eq. (3.15) by virtue of (3.15a) becomes

$$
X= \pm Y^{\prime} /\left[\varphi\left(x^{1}\right) \pm \alpha^{2}\right]^{1 / 2} .
$$

The combination $R_{2}^{2}-R_{1}^{1}$ of the Einstein equations is reduced (for $\left.T_{1}^{0}=0\right)$ due to $(2.15 c)$ and $(2.15 b)$ to the relation

$$
2 \lambda_{2}\left(T_{1}^{1}-T_{2}^{2}\right)+\partial T_{1}^{1} / \partial x^{1}=0,
$$

which is the component of the conservation laws (3.5) with $i=1$ and is satisfied by (3.3a), (3.13) and (3.12a). The combination $\left(R_{0}^{0}-R_{1}^{1}\right) h_{1}$ $+2\left(R_{0}^{0}-R_{2}^{2}\right) h_{2}$ of the Einstein equations is reduced (for $T_{1}^{0}=0$ ) to the relation

$$
h_{1}\left(T_{0}^{0}-T_{1}^{1}\right)+2 h_{2}\left(T_{0}^{0}-T_{2}^{2}\right)+\partial T_{0}^{0} / \partial \tau=0,
$$

which is the component of (3.5) with $i=0$ and is also satisfied by (3.3a), (3.13) and (3.12a).

The Eq. (2.15b) together with (3.15b), (3.3a) and (3.12a) yields after the first integration

$$
\frac{1}{c^{2}} \dot{Y}^{2}=\varphi\left(x^{1}\right)-\frac{B^{2}}{Y^{2}}+\frac{F_{1}\left(x^{1}\right)}{Y}, \quad B^{2}=\frac{k K^{2}}{c^{4}}=\text { const },
$$

with an arbitrary function $F_{1}\left(x^{1}\right)$. The further integration of (3.18) depends upon the sign of function $\varphi\left(x^{1}\right)$.

In the cases $f\left(x^{2}\right)=1$ and $f\left(x^{2}\right)=\sinh x^{2}$ with the conditions (3.17a) and (3.17b) the result of the integration of (3.18) can be given in a parametric form with a parameter $\eta$ which depends upon $\tau$ and $x^{1}$ :

$$
\begin{gathered}
c \tau=c \tau_{0}\left(x^{1}\right)+F\left(x^{1}\right)\left\{ \pm\left[1+B^{2} \chi\left(x^{1}\right)\right]^{1 / 2} \sinh \eta-\eta\right\}, \\
Y=\left[\varphi\left(x^{1}\right)\right]^{1 / 2} F\left(x^{1}\right)\left\{ \pm\left[1+B^{2} \chi\left(x^{1}\right)\right]^{1 / 2} \cosh \eta-1\right\}, \\
F\left(x^{1}\right) \equiv F_{1}\left(x^{1}\right) / 2\left[\varphi\left(x^{1}\right)\right]^{3 / 2}, \quad \chi\left(x^{1}\right)=1 / F^{2}\left(x^{1}\right) \varphi^{2}\left(x^{1}\right),
\end{gathered}
$$

where $\tau_{0}\left(x^{1}\right)$ is an arbitrary function. Two signs in (3.19) corresponds to two types of the solutions $[7,13]$. Formulae (3.19) give

$$
\begin{aligned}
Y^{\prime}= & \frac{1}{2} \frac{\varphi^{\prime}}{\varphi} Y-2 \varphi^{1 / 2} F^{\prime}-\frac{\varphi^{1 / 2} F B^{2} \chi^{\prime}}{2\left(1+B^{2} \chi\right)}+\frac{\varphi F}{Y} \\
& \cdot\left\{F^{\prime}\left[B^{2} \chi \pm\left(1+B^{2} \chi\right)^{1 / 2} \eta \sinh \eta\right] \mp\left(1+B^{2} \chi\right)^{1 / 2} c \tau_{0}^{\prime} \sinh \eta\right. \\
& \left.+F B^{4} \chi^{\prime} \chi\left[2\left(1+B^{2} \chi\right)\right]^{-1}\right\} .
\end{aligned}
$$

3 Commun math Phys, Vol 26 
For $X$ the formulae (3.15b) and (3.19a) are valid with the values (3.16), (3.17a) and (3.17b). The substitution of (3.19), (3.15b) into the equation $R_{0}^{0}-(R / 2)$ gives the expression for $e(3.13)$ in the form

$$
e=\frac{c^{4} F|\varphi|^{3 / 2}}{4 \pi k Y^{2} Y^{\prime}}\left(\frac{3}{2} \frac{\varphi^{\prime}}{\varphi}+\frac{F^{\prime}}{F}\right)=\frac{c^{4}}{8 \pi k} \frac{F_{1}^{\prime}}{Y^{2} Y^{\prime}} .
$$

(3.19), (3.15b), (3.19a), (3.20) and (3.12) give the complete solution for the values (2.2a) and (2.2b) in (2.1), (2.1a). The presence of a magnetic field manifests itself in a nonzero value of a constant $B$. The solution contains arbitrary functions $\tau_{0}\left(x^{1}\right), F\left(x^{1}\right)$ and also $\varphi\left(x^{1}\right)$ with the conditions (3.17a), (3.17b).

In the case $f\left(x^{2}\right)=\sin x^{2}$ a function $\varphi\left(x^{1}\right)$ in (3.18) may be positive, zero or negative. For $\varphi\left(x^{1}\right)>0$ Eqs. (3.19), (3.19a) and (3.15b), (3.20) remain valid with $(3.16),(3.15 b)$. For values of $\varphi\left(x^{1}\right)$ in $(3.17 \mathrm{c})$ in the interval

$$
-1<\varphi\left(x^{1}\right)<0
$$

the result of the integration of (3.18) is given in a parametric form

$$
\begin{aligned}
& c \tau=c \tau_{0}\left(x^{1}\right)+F\left(x^{1}\right)\left\{\eta-\left[1-B^{2} \chi\left(x^{1}\right)\right]^{1 / 2} \sin \eta\right\}, \\
& Y=F\left(x^{1}\right)\left|\varphi\left(x^{1}\right)\right|^{1 / 2}\left\{1-\left[1-B^{2} \chi\left(x^{1}\right)\right]^{1 / 2} \cos \eta\right\}, \\
& F\left(x^{1}\right) \equiv F_{1}\left(x^{1}\right) / 2\left[-\varphi\left(x^{1}\right)\right]^{3 / 2}, \quad \chi\left(x^{1}\right)=1 / F^{2}\left(x^{1}\right) \varphi^{2}\left(x^{1}\right),
\end{aligned}
$$

with an arbitrary function $\tau_{0}\left(x^{1}\right)$. Formulae (3.22) give

$$
\begin{aligned}
Y^{\prime} & =\frac{1}{2} \frac{\varphi^{\prime}}{\varphi} Y+2|\varphi|^{1 / 2} F^{\prime}-\frac{|\varphi|^{1 / 2} F B^{2} \chi^{\prime}}{2\left(1-B^{2} \chi\right)}+\frac{\varphi F}{Y} \\
& \cdot\left\{F^{\prime}\left[B^{2} \chi+\left(1-B^{2} \chi\right)^{1 / 2} \eta \sin \eta\right]+\left(1-B^{2} \chi\right)^{1 / 2} c \tau_{0}^{\prime} \sin \eta\right. \\
& \left.-F B^{4} \chi^{\prime} \chi\left[2\left(1-B^{2} \chi\right)\right]^{-1}\right\} .
\end{aligned}
$$

$X$ is given by (3.15b) with the values (3.16), (3.21) and with (3.22a). For $e$ in the case (3.21) the formula (3.20) remains valid. In virtue of (3.22), (3.22a) an arbitrary function $\chi\left(x^{1}\right)$ for (3.21) must satisfy the inequality $\chi\left(x^{1}\right) \leqq 1 / B^{2}$, i.e. $F^{2} \varphi^{2} \geqq B^{2}$.

Finally, in the case $f\left(x^{2}\right)=\sin x^{2}$ for

$$
\varphi\left(x^{1}\right)=0
$$

the result of the integration of (3.18) is given by

$$
\begin{aligned}
& c \tau=c \tau_{0}\left(x^{1}\right)+\frac{1}{12} F_{1}\left(x^{1}\right) \lambda^{3}+\frac{1}{F_{1}\left(x^{1}\right)} B^{2} \lambda, \\
& Y=\frac{1}{4} F_{1}\left(x^{1}\right) \lambda^{2}+\frac{1}{F_{1}\left(x^{1}\right)} B^{2} .
\end{aligned}
$$


Eqs. (3.24) and (3.15b), (3.16), (3.23) give also

$$
\begin{aligned}
X & = \pm Y^{\prime}, \\
Y^{\prime} & =\frac{F_{1}^{\prime}}{3 F_{1}^{2}}\left(Y F_{1}+4 B^{2}\right)-\frac{1}{2 Y}\left[\frac{16}{3} B^{4} \frac{F_{1}^{\prime}}{F_{1}^{3}}+\lambda F_{1} c \tau_{0}^{\prime}\left(x^{1}\right)\right], \\
e & =\frac{c^{4}}{8 \pi k} \frac{F_{1}^{\prime}}{Y^{2} Y^{\prime}} .
\end{aligned}
$$

The energy density of a magnetic field $W$ is given by (3.12a). If a magnetic field is present $(B \neq 0)$, then in all possible cases due to (3.19), (3.22) and (3.24) a value of $Y$ never is reduced to zero. So $W$ remains finite for all moments of time. The moment $\tau^{*}$ when $X$ becomes zero, being obtained from (3.15b), (3.19a), (3.22a) and (3.24a), corresponds due to (3.20), (3.15b) to a singularity with $e=\infty$. In a general case of a metric (2.1a) with groups $G_{3}$ a singular state is achieved for various points $x^{1}$ at various moments $\tau^{*}$. Near a singular state $\eta=\eta^{*}\left(x^{1}\right), \tau=\tau^{*}\left(x^{1}\right)$ the dependence of $X$ and $Y$ upon $\tau$ has a structure

$$
X=\left[\tau-\tau^{*}\left(x^{1}\right)\right] F_{2}\left(x^{1}\right), \quad Y=Y^{*}\left(x^{1}\right)+\left(\tau-\tau^{*}\right) F_{3}\left(x^{1}\right) .
$$

For $f\left(x^{2}\right)=\sin x^{2}$ and (3.21) the dependence of $Y$ upon $\tau$ due to (3.22) has an oscillating character with a limited amplitude, while an amplitude of an oscillating function $X$ of $\tau$ increases with increasing time. In the cases $f\left(x^{2}\right)=\sinh x^{2}$ and $f\left(x^{2}\right)=1$ and also in the case $f\left(x^{2}\right)=\sin x^{2}$ with $\varphi \geqq 0$ values of $X$ and $Y$ approach infinity asymptotically as $|\tau| \rightarrow \infty$ in the following way

$$
\begin{array}{ll}
\varphi>0: Y \approx\left[\varphi\left(x^{1}\right)\right]^{1 / 2} c \tau, & X \approx Y \varphi^{\prime} / 2 \varphi\left(\varphi \pm \alpha^{2}\right)^{1 / 2}, \\
\varphi=0: Y \approx\left(9 F_{1} / 4\right)^{1 / 3}(c \tau)^{2 / 3}, & X \approx \pm Y F_{1}^{\prime} / 3 F_{1} .
\end{array}
$$

The non-central extensions of algebras $A_{3}$ of the groups $G_{3}$ keep the condition $\lambda_{2} \neq 0$. The noncentral extension of $A_{3}$ to $A_{4}$ for $f\left(x^{2}\right)=1$ with the metric (2.9a) corresponds to a homogeneous axially symmetric model with a co-moving 3-space of constant negative curvature in accordance with (2.16). For such a metric an energy density of a magnetic field in virtue of $(2.15 b)$ would depend only upon $\tau$ in the contradiction with (3.12a) and (2.9a). Thus, such a non-central extension becomes impossible when a magnetic field is present ${ }^{1}$. If only matter is present $(B=0)$, such an extension is possible, and in this case in the system with (2.9a) one obtains that $R_{1}^{0} \neq 0$ and $u^{1}(\tau) \neq 0$ in (3.4). The integration becomes possible after a transformation from (2.9a) into a synchronous and co-moving system with a metric (2.1), (2.1a) [21], and the result is given by

1 The fact that homogeneous Bianchi type $\mathrm{V}$ space-times cannot admit a magnetic field is shown in [25]. 
(3.19), (3.19a), (3.15b), (3.20) with the values of functions

$\varphi\left(x^{1}\right)=\exp \left(-2 x^{1}\right), F=$ const, $\tau_{0}\left(x^{1}\right)=K_{0} x^{1}+$ const, $K_{0}=$ const $; B=0$.

In the case when only matter is present $(B=0)$ the further extensions to $G_{6}$, which lead to Friedmann models, are possible with a certain choice of arbitrary functions in the solutions. For (2.2a) one obtains from (3.19), (3.19a) the open Friedmann model with (2.10) for $F=$ const, $\tau_{0}=$ const and

$$
f\left(x^{2}\right)=1: \varphi\left(x^{1}\right)=\exp \left(-2 x^{1}\right) .
$$

For (2.2b) one obtains in (3.19) the open Friedmann model with (2.11) for $F=$ const, $\tau_{0}=$ const and

$$
f\left(x^{2}\right)=\sinh x^{2}: \varphi\left(x^{1}\right)=\cosh ^{2} x^{1} .
$$

For (2.2c) one comes in (3.19) to the open Friedmann model if $F=$ const, $\tau_{0}=$ const and

$$
f\left(x^{2}\right)=\sin x^{2}: \varphi\left(x^{1}\right)=\sinh ^{2} x^{1},
$$

obtains the flat Friedmann model in (3.24) if $\tau_{0}=$ const and

$$
f\left(x^{2}\right)=\sin x^{2}: \varphi\left(x^{1}\right)=0, \quad F_{1}\left(x^{1}\right)=\operatorname{const}\left(x^{1}\right)^{3},
$$

and has in (3.22) the closed Friedmann model if $\varphi\left(x^{1}\right)=-\sin ^{2} x^{1}$, $F=$ const, $\tau_{0}=$ const.

In the case when matter and a magnetic field are present for the groups $G_{3}$ the choice of arbitrary functions in the solutions with $\varphi\left(x^{1}\right) \geqq 0$ is possible for which the solutions become asymptotically isotropic as $|\tau| \rightarrow \infty$.

For $\varphi>0$ according to (3.25) such solutions, which approach the open Friedmann model as $|\tau| \rightarrow \infty$, are given by (3.19), (3.19a) with the functions $\varphi\left(x^{1}\right)$ which are chosen due to formulae (3.27), (3.28), (3.29) and with arbitrary functions $F_{1}\left(x^{1}\right)$ and $\tau_{0}\left(x^{1}\right)$.

For $\varphi=0$ according to (3.26) the formulae (3.24), (3.24a) give solutions which approach with $|\tau| \rightarrow \infty$ the flat Friedmann model for $F_{1}\left(x^{1}\right)$ being chosen due to (3.30) and for an arbitrary $\tau_{0}\left(x^{1}\right)$.

In the case when the signature of $V_{2}$ is $(+-)$ and a metric has a form $(2.17 \mathrm{a}),(2.17 \mathrm{~b})$, the construction of gravitational fields with matter becomes impossible since the invariance of the 4-velocity with respect to the transformations with (2.18)-(2.21) would lead to a physically senseless condition of vanishing of zero component of the 4-velocity. Nevertheless, in this case gravitational fields may be considered the sources of which are collinear magnetic and electric fields directed along $x^{1}$ with the energy-momentum tensor given by (3.12a). Calculations 
similar to the previous give for the metric (2.17a)

$$
X= \pm\left[\mp \alpha^{2}-\varphi\left(x^{2}\right)\right]^{-1 / 2} \partial Y / \partial x^{2}, \quad \mp \alpha^{2}=d^{2} f\left(x^{0}\right) / f\left(d x^{0}\right)^{2},
$$

and for the metric (2.17b)

$$
X= \pm\left[\mp \alpha^{2}+\varphi\left(x^{2}\right)\right]^{-1 / 2} \partial Y / \partial x^{2}, \quad \mp \alpha^{2}=d^{2} f\left(x^{1}\right) / f\left(d x^{1}\right)^{2},
$$

with an arbitrary function $\varphi\left(x^{2}\right)$, which obeys corresponding inequalities. In both cases (2.17a) (2.17b) the expressions for $Y$ are given by $(A=$ const)

$$
\begin{aligned}
\varphi>0: x^{3} & =\Phi\left(x^{2}\right)+\left[\varphi\left(x^{2}\right)\right]^{-3 / 2}\left[ \pm\left(A^{2}+B^{2} \varphi\right)^{1 / 2} \sinh \eta-A \eta\right], \\
Y & =\left[\varphi\left(x^{2}\right)\right]^{-1}\left[ \pm\left(A^{2}+B^{2} \varphi\right)^{1 / 2} \cosh \eta-A\right] ; \\
\varphi<0: \quad x^{3} & =\Phi\left(x^{2}\right)+\left|\varphi\left(x^{2}\right)\right|^{-3 / 2}\left[A \eta-\left(A^{2}-B^{2} \varphi\right)^{1 / 2} \sin \eta\right], \\
Y & =\left|\varphi\left(x^{2}\right)\right|^{-1}\left[A-\left(A^{2}-B^{2} \varphi\right)^{1 / 2} \cos \eta\right] ; \\
\varphi=0: \quad x^{3} & =\Phi\left(x^{2}\right)+\frac{\lambda\left(A^{2} \lambda^{2}+3 B^{2}\right)}{6 A}, Y=\frac{A^{2} \lambda^{2}+B^{2}}{2 A}, X=\mp \frac{\lambda A \Phi^{\prime}\left(x^{2}\right)}{Y},
\end{aligned}
$$

where $\Phi\left(x^{2}\right)$ is an arbitrary function.

In the case of the group $G_{4}$ with (2.17a), (2.17b), (2.21) the solution for $f=1$ is given by [7]

$$
Y=\left(A^{2} \eta^{2}+B^{2}\right) / 2 A, \quad x^{3}=\eta\left(A^{2} \eta^{2}+3 B^{2}\right) / 6 A, \quad X=\text { const } \eta / Y \text {. }
$$

For $f\left(x^{0}\right)=\sinh x^{0}$ in (2.17a), (2.21) and for $f\left(x^{1}\right)=\sin x^{1}$ in (2.17b), (2.21) the solution is

$$
\begin{gathered}
Y= \pm\left(A^{2}+B^{2}\right)^{1 / 2} \cosh \eta-A, \quad x^{3}= \pm\left(A^{2}+B^{2}\right)^{1 / 2} \sinh \eta-A \eta, \\
X=\text { const } \sinh \eta / Y .
\end{gathered}
$$

For $f\left(x^{0}\right)=\sin x^{0}$ in (2.17a), (2.21) and for $f\left(x^{1}\right)=\sinh x^{1}$ in (2.17b), (2.21) the solution is given by

$$
\begin{gathered}
Y=A-\left(A^{2}-B^{2}\right)^{1 / 2} \cos \eta, \quad x^{3}=A \eta-\left(A^{2}-B^{2}\right)^{1 / 2} \sin \eta, \\
X=\mathrm{const} \sin \eta / Y .
\end{gathered}
$$

For the metric (2.17b) one thus obtains the static solutions for magneto-gravitational configurations. In this case formulae (3.31) and (3.32) with $A=B$ are reduced to previously studied solutions [26, 27].

\section{References}

1. Taub, A.H.: Ann. Math. 53, 472 (1951).

2. Petrov, A.Z.: Einstein spaces. New York: Pergamon 1969.

3. Landau, L.D., Lifshitz,E. M.: Field theory. Moscow: Nauka 1967.

4. Bondi, H.: Monthly notices roy. Astron. Soc. 107, 410 (1947). 
5. Zeldovič, Ya. B.: Zh. Eksp. Teor. Fiz. 48, 986 (1965); - Sov. Phys. JETP 21, 656(1965).

6. Doroshkevich, A. G.:Astrofizika 1, 255 (1965), in Russian.

7. Shikin, I. S.: Dokl. Akad. Nauk SSSR 171, 73 (1966); - Sov. Phys. Dokl. 11, 944 (1967).

8. - Dokl. Akad. Nauk SSSR 176, 1048 (1967); - Sov. Phys. Dokl. 12, 950 (1968).

9. Thorne, K. S.: Astrophys. J. 148, 51 (1967).

10. Jacobs, K.C.: Astrophys. J. 155, 379 (1969).

11. Goenner, H.: Commun. math. Phys. 16, 34 (1970).

12. Kompaneets, A.S., Chernov,A.S.: Zh. Eksp. Teor. Fiz. 47, 1939 (1964); - Sov. Phys. JETP 20, 1303 (1965).

13. Kantowski, R., Sachs, R. K.: J. Math. Phys. 7, 443 (1966).

14. Estabrook, F. B., Wahlquist, H. D., Behr, C. G.: J. Math. Phys. 9, 497 (1968).

15. Goenner, H., Stachel, J.: J. Math. Phys. 11, 3358 (1970).

16. Ellis, G.F.R.: J. Math. Phys. 8, 1171 (1967).

17. Stewart, J.M., Ellis, G. F. R.: J. Math. Phys. 9, 1072 (1968).

18. Vajk, J.P., Eltgroth, P. G.: J. Math. Phys. 11, 2212 (1970).

19. Chap. 11 of Gravitation: An introduction to current research, ed. by L. Witten. New York: Wiley 1962.

20. Ellis, G.F.R., MacCallum, M. A.H.: Commun. math. Phys. 12, 108 (1969).

21. Farnsworth, D. L.: J. Math. Phys. 8, 2315 (1967).

22. Lichnerowicz,A.: Relativistic hydrodynamics and magneto-hydrodynamics. New York: Benjamin 1967.

23. Shikin, I. S.: Ann. Inst. Henri Poincaré A 11, 343 (1969).

24. Ruban, V. A.: Zh. Eksp. Teor. Fiz. 56, 1914 (1969); - Sov. Phys. JETP 29, 1027 (1969).

25. Hughston, L.P., Jacobs, K. C.: Astrophys. J. 160, 147 (1970).

26. Melvin, M. A.: Phys. Rev. 139. B 225 (1965).

27. Bertotti, B.: Phys. Rev. 116, B 1331 (1959).

Robinson, I.: Bull. Acad. Polon. Sci. Ser. Math. 7, 351 (1959).

I. S. Shikin

Moscow State University

Michurinsky prospect, 1

Moscow B 234, USSR 